\title{
Domination Polynomials of $\boldsymbol{k}$-Tree Related Graphs
}

\author{
Somayeh Jahari and Saeid Alikhani \\ Department of Mathematics, Yazd University, Yazd 89195-741, Iran \\ Correspondence should be addressed to Saeid Alikhani; alikhani206@gmail.com
}

Received 27 August 2014; Accepted 21 October 2014; Published 11 November 2014

Academic Editor: Johannes Hendrik Hattingh

Copyright (C) 2014 S. Jahari and S. Alikhani. This is an open access article distributed under the Creative Commons Attribution License, which permits unrestricted use, distribution, and reproduction in any medium, provided the original work is properly cited.

Let $G$ be a simple graph of order $n$. The domination polynomial of $G$ is the polynomial $D(G, x)=\sum_{i=\gamma(G)}^{n} d(G, i) x^{i}$, where $d(G, i)$ is the number of dominating sets of $G$ of size $i$ and $\gamma(G)$ is the domination number of $G$. In this paper, we study the domination polynomials of several classes of $k$-tree related graphs. Also, we present families of these kinds of graphs, whose domination polynomials have no nonzero real roots.

\section{Introduction}

Throughout this paper, we will consider only simple graphs. Let $G=(V, E)$ be a simple graph. For $F \subseteq V(G)$ we use $\langle F\rangle$ for the subgraph induced by $F$. For any vertex $v \in V(G)$, the open neighborhood of $v$ is the set $N(v)=\{u \in V(G) \mid$ $\{u, v\} \in E(G)\}$ and the closed neighborhood of $v$ is the set $N[v]=N(v) \cup\{v\}$. For a set $S \subseteq V(G)$, the open neighborhood of $S$ is $N(S)=\bigcup_{v \in S} N(v)$ and the closed neighborhood of $S$ is $N[S]=N(S) \cup S$. For every vertex $v \in V(G)$, the degree of $v$ is the number of edges incident with $v$ and is denoted by $d_{G}(v)=|N(v)|$. Let $d_{i}, 1 \leq i \leq n$, be the degrees of the vertices $v_{i}$ of a graph in any order. The sequence $\left\{d_{i}\right\}_{1}^{n}$ is called the degree sequence of the graph. A clique in a graph is a subset of its vertices such that every two vertices in the subset are connected by an edge. We use $K_{n}, P_{n}, C_{n}$, and $S_{1, n-1}$ for a clique, a path, a cycle, and a star, all of order $n$, respectively.

A set $S \subseteq V(G)$ is a dominating set if $N[S]=V$ or, equivalently, every vertex in $V(G) \backslash S$ is adjacent to at least one vertex in $S$. The domination number $\gamma(G)$ is the minimum cardinality of a dominating set in $G$. A dominating set with cardinality $\gamma(G)$ is called a $\gamma$-set. For a detailed treatment of these parameters, the reader is referred to [1]. Let $\mathscr{D}(G, i)$ be the family of dominating sets of a graph $G$ with cardinality $i$ and let $d(G, i)=|\mathscr{D}(G, i)|$. The domination polynomial $D(G, x)$ of $G$ is defined as $D(G, x)=\sum_{i=\gamma(G)}^{|V(G)|} d(G, i) x^{i}$, where $\gamma(G)$ is the domination number of $G$ (see $[2,3])$. Thus, $D(G, x)$ is the generating polynomial for the number of dominating sets of $G$ of any cardinality. A root of $D(G, x)$ is called a domination root of $G$.

In [4], it is shown that computing the domination polynomial $D(G, x)$ of a graph $G$ is NP-hard and some examples for graphs for which $D(G, x)$ can be computed efficiently are given. The vertex contraction $G / u$ of a graph $G$ by a vertex $u$ is the operation under which all vertices in $N(u)$ are pairwise joined to each other and then $u$ is deleted (see [5]). The following theorem is useful for finding the recurrence relations for the domination polynomials of arbitrary graphs.

Theorem 1 (see $[4,6])$. Let $G$ be a graph. For any vertex $u$ in $G$, one has

$$
\begin{aligned}
D(G, x)= & x D\left(\frac{G}{u}, x\right)+D(G-u, x)+x D(G-N[u], x) \\
& -(1+x) p_{u}(G, x),
\end{aligned}
$$

where $p_{u}(G, x)$ is the polynomial counting the dominating sets of $G-u$ which do not contain any vertex of $N(u)$ in $G$.

Using Theorem 1 we are able to obtain an easier formula for a graph with at least one vertex of degree 1 . Since every tree has at least two vertices of degree 1 , we can use the following recurrence to obtain the domination polynomials of trees. 

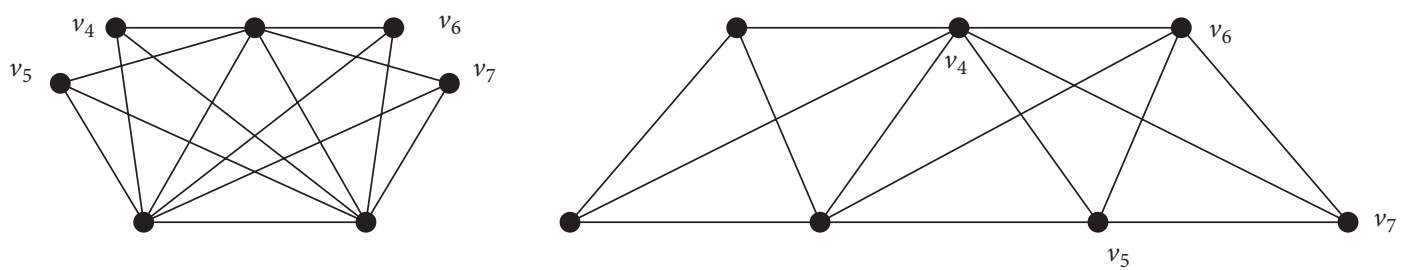

FIGURE 1: The 3-star and 3-path on 7 vertices, respectively.

Corollary 2 (see [4]). Let $G=(V, E)$ be a graph, let $v$ be a vertex of degree 1 in $G$, and let $u$ be its neighbor. Then,

$$
\begin{aligned}
D & (G, x) \\
& =x\left(D\left(\frac{G}{u}, x\right)+D(G-u-v, x)+D(G-N[u], x)\right) .
\end{aligned}
$$

If $G_{1}$ and $G_{2}$ are disjoint graphs of orders $n_{1}$ and $n_{2}$, respectively, then $D\left(G_{1} \cup G_{2}, x\right)=D\left(G_{1}, x\right) D\left(G_{2}, x\right)$ and

$$
\begin{aligned}
D\left(G_{1}+G_{2}, x\right)= & \left((1+x)^{n_{1}}-1\right)\left((1+x)^{n_{2}}-1\right) \\
& +D\left(G_{1}, x\right)+D\left(G_{2}, x\right),
\end{aligned}
$$

where $G_{1}+G_{2}$ is the join of $G_{1}$ and $G_{2}$, formed from $G_{1} \cup G_{2}$ by adding in all edges between a vertex of $G_{1}$ and a vertex of $G_{2}$ (see [2]).

The domination polynomials of trees, aside from path graph, have not been studied and there is no study for coefficients of $D(T, x)$ for trees $T$ with $n$ vertices. In this paper, $k$-trees, a generalization of trees, are considered. We study, similar to [7], $k$-tree related graphs and study their domination polynomials. An interesting study of the roots of domination polynomials is done in $[8,9]$. Classification of the roots of domination polynomials is difficult to do, as well as finding graphs with no nonzero real roots. In this paper, we present $k$-tree related families which have this prementioned property.

In Section 2, we study the domination polynomials for some $k$-tree related graphs. In Section 3, we present some families of these kinds of graphs whose domination polynomials have no nonzero real roots.

\section{Domination Polynomials of $k$-Tree Related Graphs}

In this section, we study the domination polynomials for some $k$-tree related graphs. The class of $k$-trees is a very important subclass of triangulated graphs. Harary and Palmer [10] first introduced 2-trees in 1968. Beineke and Pippert [11] gave the definition of a $k$-tree in 1969. In the literature on $k$-trees, there are interesting applications to the study of computational complexity.

Definition 3. For a positive integer $k$, a $k$-tree, denoted by $T_{n}^{k}$, is defined recursively as follows. The smallest $k$-tree is the $k$ clique $K_{k}$. If $G$ is a $k$-tree with $n \geq k$ vertices and a new vertex $v$ of degree $k$ is added and joined to the vertices of a $k$-clique in $G$, then the larger graph is a $k$-tree with $n+1$ vertices.

An independent set in a graph $G$ is a set of pairwise nonadjacent vertices.

Definition 4. Let $K_{k}$ be a $k$-clique and let $S$ be an independent set of $n-k$ vertices. A $(k, n)$-star, denoted by $S_{k, n-k}$, is defined as $S_{k, n-k}=K_{k}+S$.

Definition 5. A $(k, n)$-path, denoted by $P_{n}^{k}$, begins with $k$ clique on $\left\{v_{1}, v_{2}, \ldots, v_{k}\right\}$. For $i=k+1$ to $n$, let vertex $v_{i}$ be adjacent to vertices $\left\{v_{i-1}, v_{i-2}, \ldots, v_{i-k}\right\}$ only (see Figure 1 ).

A helpful characteristic of the $(k, n)$-path $P_{n}^{k}$ is that we may order the vertices $v_{1}, v_{2}, \ldots, v_{n}$ such that $P_{n}^{k} \backslash\left\{v_{1}, \ldots, v_{i}\right\}$ is a $k$-path on $n-i$ vertices for $1 \leq i \leq n-k-1$; such a vertex ordering is referred to as a presentation.

Definition 6. A $(k, n)$-cycle, denoted by $C_{n}^{k}$, consists of a $(k, n)$-path on $\left\{v_{1}, v_{2}, \ldots, v_{n}\right\}$ defined as above and an edge joining $v_{1}$ to $v_{n}$, where $n \geq k+2$.

Definition 7. If $G$ is a $(k, n)$-cycle of order $n$ and $v$ is a vertex not in $G$, then $G+v$ is called a $(k, n)$-wheel and is denoted by $W_{n}^{k}$

Notice that $P_{n}^{1}, C_{n}^{1}, W_{n}^{1}$, and $S_{1, n-1}$ are just the standard path, cycle, wheel, and star, respectively. It follows easily from the domination polynomial of join of two graphs that, for the star graph $S_{1, n-1}$, we have $D\left(S_{1, n-1}, x\right)=x(1+x)^{n-1}+x^{n-1}$. The following are recurrences for the domination polynomials of paths and cycles [12].

Theorem 8. For the natural number $n \geq 3$,

(i) $D\left(P_{n+1}, x\right)=x\left(D\left(P_{n}, x\right)+D\left(P_{n-1}, x\right)+D\left(P_{n-2}, x\right)\right)$, where $D\left(P_{1}, x\right)=x, D\left(P_{2}, x\right)=x^{2}+2 x$, and $D\left(P_{3}, x\right)=x^{3}+3 x^{2}+x$;

(ii) $D\left(C_{n+1}, x\right)=x\left(D\left(C_{n}, x\right)+D\left(C_{n-1}, x\right)+D\left(C_{n-2}, x\right)\right)$, where $D\left(C_{1}, x\right)=x, D\left(C_{2}, x\right)=x^{2}+2 x$, and $D\left(C_{3}, x\right)=x^{3}+3 x^{2}+3 x$.

Note that both $(k, n)$-cycles and $(k, n)$-wheels are not $k$ trees. But they are closely related to $k$-trees. We begin with a simple lemma which was proven in [7] as Proposition 2. 
Lemma 9. For any $k$-tree $T_{n}^{k},\left|E\left(T_{n}^{k}\right)\right|=k n-(1 / 2) k(k+1)=$ $\left(2 n k-k^{2}-k\right) / 2$.

The independence number is the size of a maximum independent set in a graph and is denoted by $\alpha(G)$. The following lemma gives independence numbers for $k$-tree related graphs.

Lemma 10 (see [7]). For each natural number $k \leq n$, one has

(i) $\alpha\left(P_{n}^{k}\right)=\lfloor(n+k) /(k+1)\rfloor$,

(ii) $\alpha\left(C_{n}^{k}\right)=\lfloor(n+k-1) /(k+1)\rfloor$,

(iii) $\alpha\left(W_{n}^{k}\right)=\alpha\left(C_{n}^{k}\right)$,

(iv) $\alpha\left(S_{k, n-k}\right)=n-k$.

Now, we present the following domination numbers for $k$-tree related graphs.

Theorem 11. For each natural number $k \leq n$, one has

(i) $\gamma\left(P_{n}^{k}\right)=\lceil n /(2 k+1)\rceil$,

(ii) $\gamma\left(C_{n}^{k}\right)=\lceil n /(2 k+1)\rceil$,

(iii) $\gamma\left(W_{n}^{k}\right)=1$,

(iv) $\gamma\left(S_{k, n-k}\right)=1$.

Proof. (i) Since $k \leq n$, by the definition of $P_{n}^{k}$, the degree sequence in this graph is

$$
\{k, k+1, \ldots, 2 k-1,2 k, \ldots, 2 k, 2 k-1, \ldots, k+1, k\} ;
$$

we have $\gamma\left(P_{n}^{k}\right)=1$ for $n \leq 2 k+1$ and $\gamma\left(P_{n}^{k}\right)=2$ for $2 k+2 \leq$ $n \leq 4 k+2$. Thus, (i) holds for $k \leq n \leq 4 k+2$. Now, assume $n \geq 4 k+3$. We use induction on $n$. Since any $\gamma$-set of $P_{n}^{k}$ contains only one vertex of the $\left\{v_{n}, v_{n-1}, \ldots, v_{n-k}, \ldots, v_{n-2 k-1}\right\}$ and $P_{n}^{k}-\left\{v_{n}, v_{n-1}, \ldots, v_{n-k}, \ldots, v_{n-2 k-1}\right\}$ is a $k$-path with $n-$ $2 k-1$ vertices, by induction, $\gamma\left(P_{n}^{k}\right)=1+\gamma\left(P_{n-2 k-1}^{k}\right)=1+$ $\lceil(n-2 k-1) /(2 k+1)\rceil=\lceil n /(2 k+1)\rceil$. Hence, (i) holds.

(ii) Since $k \leq n$, by the definition of $C_{n}^{k}$, the degree sequence in this graph is

$$
\begin{gathered}
\{k+1, k+1, k+2, \ldots, 2 k-1,2 k, \ldots, \\
2 k, 2 k-1, \ldots, k+1, k+1\}
\end{gathered}
$$

we have $\gamma\left(C_{n}^{k}\right)=1$ for $n \leq 2 k+1$ and $\gamma\left(C_{n}^{k}\right)=2$ for $2 k+2 \leq$ $n \leq 4 k+2$. Thus, (ii) holds for $k \leq n \leq 4 k+2$. Now, assume $n \geq 4 k+3$ and use induction on $n$. Since any $\gamma$-set of $C_{n}^{k}$ contains only one vertex of the $\left\{v_{n}, v_{n-1}, \ldots, v_{n-k}, \ldots, v_{n-2 k-1}\right\}$ and $C_{n}^{k}-\left\{v_{n}, v_{n-1}, \ldots, v_{n-k}, \ldots, v_{n-2 k-1}\right\}$ is a $k$-path with $n-$ $2 k-1$ vertices, by induction, $\gamma\left(C_{n}^{k}\right)=1+\gamma\left(P_{n-2 k-1}^{k}\right)=1+$ $\lceil(n-2 k-1) /(2 k+1)\rceil=\lceil n /(2 k+1)\rceil$. Hence, (ii) holds.

(iii) Since the $(k, n)$-wheel $W_{n}^{k}$ has a vertex $v$ of degree $n-$ 1, (iii) holds.

(iv) Since the $k$-star graph $S_{k, n-k}$ has $k$ vertices of degree $n-1$, (iv) holds.

The following theorem gives a recurrence formula for the domination polynomial of $(k, n)$-path graphs.
Theorem 12. If $n \leq k+1$, then $D\left(P_{n}^{k}, x\right)=D\left(K_{n}, x\right)$. For every $k+2 \leq n$,

$$
\begin{aligned}
D\left(P_{n}^{k}, x\right)= & (1+x) D\left(P_{n-1}^{k}, x\right) \\
& +x D\left(P_{n-k-1}^{k}, x\right)-(1+x) p_{u}\left(P_{n}^{k}, x\right),
\end{aligned}
$$

where

$$
p_{u}\left(P_{n}^{k}, x\right)=\left\{\begin{array}{c}
x(1+x)^{n-k-2} \\
k+2 \leq n \leq 2 k+2 \\
x\left((1+x)^{n-k-2}-(1+x)^{n-2 k-3}\right) \\
2 k+3 \leq n \leq 2 k+6, \\
p_{u}\left(P_{n}^{k}, x\right) \\
2 k+7 \leq n .
\end{array}\right.
$$

Proof. If $n \leq k+1$, then $P_{n}^{k} \cong K_{n}$. For every $k+2 \leq$ $n$, we use Theorem 1 for the last vertex of $P_{n}^{k}$ and since (by the definition of $P_{n}^{k}$ ) the first $k+1$ and the last $k+$ 1 vertices form two cliques, we have $P_{n}^{k} / u \cong P_{n}^{k}-u$. It is clear that $D\left(P_{n}^{k}-N[u], x\right)=D\left(P_{n-k-1}^{k}, x\right)$. Obviously, $p_{u}\left(P_{n}^{k}, x\right)$ is the polynomial counting the dominating sets of $P_{n-k-1}^{k}$ and contains the vertex $v_{n-k-1}$. However, finding this polynomial involves complex calculations. We therefor give this polynomial only for $n \leq 2 k+6$. Therefore, we have the result.

In general, finding the domination polynomial of a graph is a very difficult problem. In [4], Kotek et al. showed that there exist recurrence relations for the domination polynomial which allow for efficient schemes to compute the polynomial for some types of graphs. Consider the $(k, n)$ cycle graphs. If $n \leq k+2$, then $C_{n}^{k} \cong K_{n}$. Consequently, in this case, $D\left(C_{n}^{k}, x\right)=D\left(K_{n}, x\right)$. Until now, for every $k+3 \leq n$, all attempts to find formulas for $D\left(C_{n}^{k}, x\right)$ have failed.

The following theorem gives a formula for the domination polynomial of $(k, n)$-wheel graphs.

Theorem 13. For $a(k, n)$-wheel $W_{n}^{k}$, one has

$$
D\left(W_{n}^{k}, x\right)=x(1+x)^{n}+D\left(C_{n}^{k}, x\right) .
$$

Proof. Since $W_{n}^{k}=C_{n}^{k}+K_{1}$, then

$$
\begin{aligned}
D\left(W_{n}^{k}, x\right) & =((1+x)-1)\left((1+x)^{n}-1\right)+x+D\left(C_{n}^{k}, x\right) \\
& =x(1+x)^{n}+D\left(C_{n}^{k}, x\right) .
\end{aligned}
$$

The following theorem gives a formula for the domination polynomial of $k$-star graphs, which is derived from the fact that the $k$-star graph is the join of complete graph $K_{k}$ and independent set $S$ (empty graph $O_{n-k}$ ).

Theorem 14. For every $k \in \mathbb{N}$ and $n>k$,

$$
D\left(S_{k, n-k}, x\right)=(1+x)^{n-k}\left((1+x)^{k}-1\right)+x^{n-k} .
$$


Proof. Let $S_{k, n-k}$ be the $k$-star graph with vertex set $V\left(S_{k, n-k}\right)=\left\{v_{1}, v_{2}, \ldots, v_{k}, v_{k+1}, \ldots, v_{n}\right\}$. It suffices to show that every dominating set of size $j=1, \ldots, n$ is accounted for exactly once in the above statement. Clearly, every nonempty subset of $\left\{v_{1}, v_{2}, \ldots, v_{k}\right\}$ is a dominating set of $k$ star graphs. These sets can be extended with any number of vertices $\left\{v_{k+1}, \ldots, v_{n}\right\}$. Also, obviously, the set $\left\{v_{k+1}, \ldots, v_{n}\right\}$ is a dominating set of $k$-star graphs. It is easy to see that there is no other method to form a dominating set for $k$-star graphs. Therefore, we have the result.

Remark 15. It is easy to see that another approach for proving Theorem 14 is using the formula for the domination polynomial of join of two graphs.

The value of a graph polynomial at a specific point can give sometimes a surprising information about the structure of the graph $[3,13]$. The following simple results give the domination polynomial of $k$-tree related graphs at -1 .

Corollary 16. For each natural number $k \leq n$, the following hold:

(i) $D\left(P_{n}^{k},-1\right)=(-1)^{\alpha\left(P_{n}^{k}\right)}$,

(ii) $D\left(W_{n}^{k},-1\right)=D\left(C_{n}^{k},-1\right)$,

(iii) $D\left(S_{k, n-k},-1\right)=(-1)^{\alpha\left(S_{k, n-k}\right)}$.

Proof. (i) Using the domination polynomial of a $(k, n)$-path in Theorem 12, for $k \leq n \leq k+1$, yields $D\left(P_{n}^{k},-1\right)=$ $D\left(K_{n},-1\right)=-1$. For every $k+2 \leq n, D\left(P_{n}^{k},-1\right)=$ $-D\left(P_{n-k-1}^{k},-1\right)$. We use induction on $n$. Suppose that the statement is true for every $k$-path with $n-k$ vertices; by induction and Lemma 10 ,

$$
\begin{aligned}
D\left(P_{n}^{k},-1\right) & =-D\left(P_{n-k-1}^{k},-1\right) \\
& =-(-1)^{\alpha\left(P_{n-k-1}^{k}\right)}=-(-1)^{\lfloor(n-1) /(k+1)\rfloor} \\
& =(-1)^{1+\lfloor(n-1) /(k+1)\rfloor}=(-1)^{\lfloor(n+k) /(k+1)\rfloor} .
\end{aligned}
$$

Hence, (i) holds.

(ii) Follows from Theorem 13.

(iii) Follows from Theorem 14 and Lemma 10.

\section{Some Families of Graphs with No Nonzero Real Domination Roots}

In [8], the authors asked the question: "Which graphs have no nonzero real domination roots?"

In this section, we obtain more results related to this question. We need some preliminaries.

For two graphs $G=(V, E)$ and $H=(W, F)$, the corona $G \circ H$ is the graph arising from the disjoint union of $G$ with $|V|$ copies of $H$, by adding edges between the $i$ th vertex of $G$ and all vertices of $i$ th copy of $H$ [14]. It is easy to see that the corona operation of two graphs does not have the commutative property.

We need the following theorem which will be used to find the domination polynomial of the corona products of two graphs.
Theorem 17 (see $[4,15])$. Let $G=(V, E)$ and $H=(W, F)$ be nonempty graphs of orders $n$ and $m$, respectively. Then,

$$
D(G \circ H, x)=\left(x(1+x)^{m}+D(H, x)\right)^{n} .
$$

A $k$-star, $S_{k, n-k}$, has vertex set $\left\{v_{1}, \ldots, v_{n}\right\}$ where $<\left\{v_{1}, v_{2}, \ldots\right.$, $\left.v_{k}\right\}>\cong K_{k}$ and $N\left(v_{i}\right)=\left\{v_{1}, \ldots, v_{k}\right\}$ for $k+1 \leq i \leq n$.

Now, we will discuss the roots of the domination polynomial of the $k$-star graphs.

\section{Theorem 18.}

(i) For odd natural $n$ and even natural $k$, no nonzero real number is the domination root of $S_{k, n-k}$.

(ii) For even natural $n$ and even natural $k$, there is at least one nonzero real domination root of $S_{k, n-k}$.

Proof. By Theorem 14, for every $n>k, D\left(S_{k, n-k}, x\right)=(1+$ $x)^{n-k}\left((1+x)^{k}-1\right)+x^{n-k}$. If $D\left(S_{k, n-k}, x\right)=0$, then we have

$$
(1+x)^{k}-1=-\left(\frac{x}{1+x}\right)^{n-k} .
$$

Now we prove the two cases of this theorem.

(i) First, suppose that $x \geq 0$. Obviously, equality (13) is true just for 0 , since for nonzero real number the left side of equality is positive but the right side is negative. If $-1<x<0$, then the left side of equality is negative but the right side is positive. Now, suppose that $x<-1$. In this case, the left side is greater than -1 and the right side $-(1 /(1+1 / x))^{n-k}$ is less than -1 , a contradiction. Therefore, in any cases, we have the result.

(ii) Let $x \geq 0$. Similar to Case (i), equality (13) is true only for 0 , since for nonzero real number the left side of equality is positive but the right side is negative. Now, suppose that $x<-1$. In this case, the right side is less than -1 and the left side is greater than -1 , a contradiction. It remains to consider $-1<x<0$. It is easy to see that $\lim _{x \rightarrow 0^{-}} D\left(S_{k, n-k}, x\right)<0$, and $D\left(S_{k, n-k},-1\right)=(-1)^{n-k}>0$. So $D\left(S_{k, n-k}, x\right)$ has at least one real root in $(-1,0)$.

Remark. Using Maple, we have shown the domination roots of $S_{4, n-4}$ for $5 \leq n \leq 44$ in Figure 2 .

Here, we construct a sequence of graphs, of which the domination roots are the same as the domination roots of the $k+1$-star graphs.

Theorem 19. The domination roots of every graph $H$ in the family

$$
\begin{aligned}
& \left\{G \circ S_{k, n-k},\left(G \circ S_{k, n-k}\right) \circ S_{k, n-k},\right. \\
& \left.\quad\left(\left(G \circ S_{k, n-k}\right) \circ S_{k, n-k}\right) \circ S_{k, n-k}, \ldots\right\}
\end{aligned}
$$

have the same behavior as the domination roots of $k+1$-star graphs. 


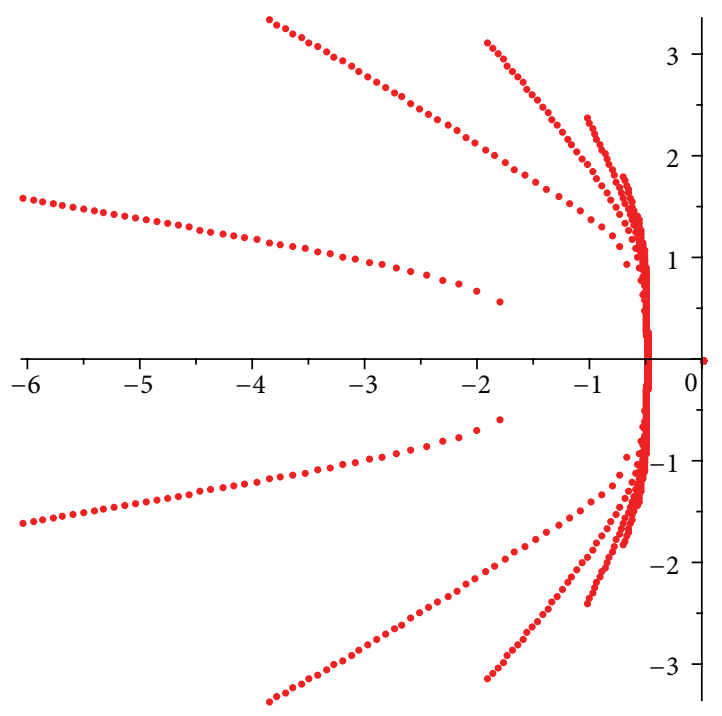

FIGURE 2: Domination roots of graphs $S_{4, n-4}$ for $5 \leq n \leq 44$.

Proof. By Theorem 17, we can deduce that, for each arbitrary graph $G$,

$$
\begin{aligned}
D & \left(G \circ S_{k, n-k}, x\right) \\
& =\left(x(1+x)^{n}+(1+x)^{n-k}\left((1+x)^{k}-1\right)+x^{n-k}\right)^{|V(G)|} \\
& =\left((1+x)^{n-k}\left((1+x)^{k+1}-1\right)+x^{n-k}\right)^{|V(G)|} \\
& =\left(D\left(S_{k+1, n-k}, x\right)\right)^{|V(G)|} .
\end{aligned}
$$

Therefore, we have the result.

\section{Conflict of Interests}

The authors declare that there is no conflict of interests regarding the publication of this paper.

\section{Acknowledgment}

The authors would like to express their gratitude to the referees for their careful reading and helpful comments.

\section{References}

[1] T. W. Haynes, S. T. Hedetniemi, and P. Slater, Fundamentals of Domination in Graphs, Marcel Dekker, New York, NY, USA, 1998.

[2] S. Akbari, S. Alikhani, and Y. H. Peng, "Characterization of graphs using domination polynomials," European Journal of Combinatorics, vol. 31, no. 7, pp. 1714-1724, 2010.

[3] S. Alikhani, "The domination polynomial of a graph at -1 ," Graphs and Combinatorics, vol. 29, no. 5, pp. 1175-1181, 2013.

[4] T. Kotek, J. Preen, F. Simon, P. Tittmann, and M. Trinks, "Recurrence relations and splitting formulas for the domination polynomial," Electronic Journal of Combinatorics, vol. 19, no. 3, p. $47,2012$.
[5] M. Walsh, "The hub number of a graph," International Journal of Mathematics and Computer Science, vol. 1, no. 1, pp. 117-124, 2006.

[6] S. Alikhani, "On the domination polynomials of non P4free graphs," Iranian Journal of Mathematical Sciences and Informatics, vol. 8, no. 2, pp. 49-55, 2013.

[7] L. Song, W. Staton, and B. Wei, "Independence polynomials of k-tree related graphs," Discrete Applied Mathematics, vol. 158, no. 8, pp. 943-950, 2010.

[8] S. Akbari, S. Alikhani, M. R. Oboudi, and Y. H. Peng, "On the zeros of domination polynomial of a graph," in Combinatorics and Graphs, vol. 531 of Contemporary Mathematics, pp. 109-115, American Mathematical Society, Providence, RI, USA, 2010.

[9] J. I. Brown and J. Tufts, "On the roots of domination polynomials," Graphs and Combinatorics, vol. 30, no. 3, pp. 527-547, 2014.

[10] F. Harary and E. M. Palmer, "On acyclic simplicial complexes," Mathematika, vol. 15, pp. 115-122, 1968.

[11] L. W. Beineke and R. E. Pippert, "The number of labeled $k$ dimensional trees," Journal of Combinatorial Theory, vol. 6, no. 2, pp. 200-205, 1969.

[12] S. Alikhani and Y.-h. Peng, "Dominating sets and domination polynomials of certain graphs. II," Opuscula Mathematica, vol. 30, no. 1, pp. 37-51, 2010.

[13] V. E. Levit and E. Mandrescu, "The independence polynomial of a graph at -1 ," http://arxiv.org/abs/0904.4819.

[14] R. Frucht and F. Harary, "On the corona of two graphs," Aequationes Mathematicae, vol. 4, pp. 322-325, 1970.

[15] S. Alikhani, "On the domination polynomial of some graph operations," ISRN Combinatorics, vol. 2013, Article ID 146595, 3 pages, 2013. 


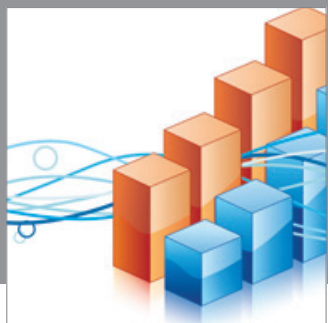

Advances in

Operations Research

mansans

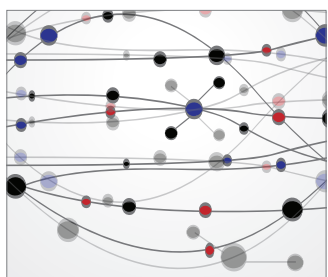

The Scientific World Journal
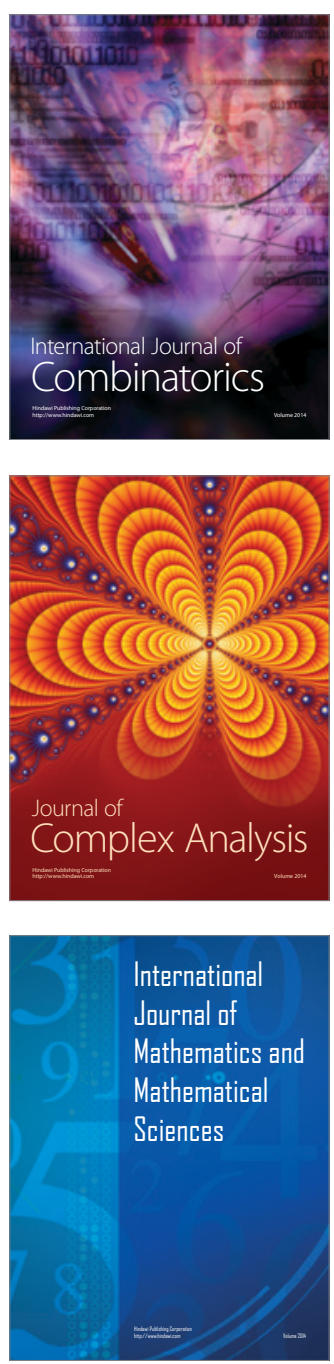
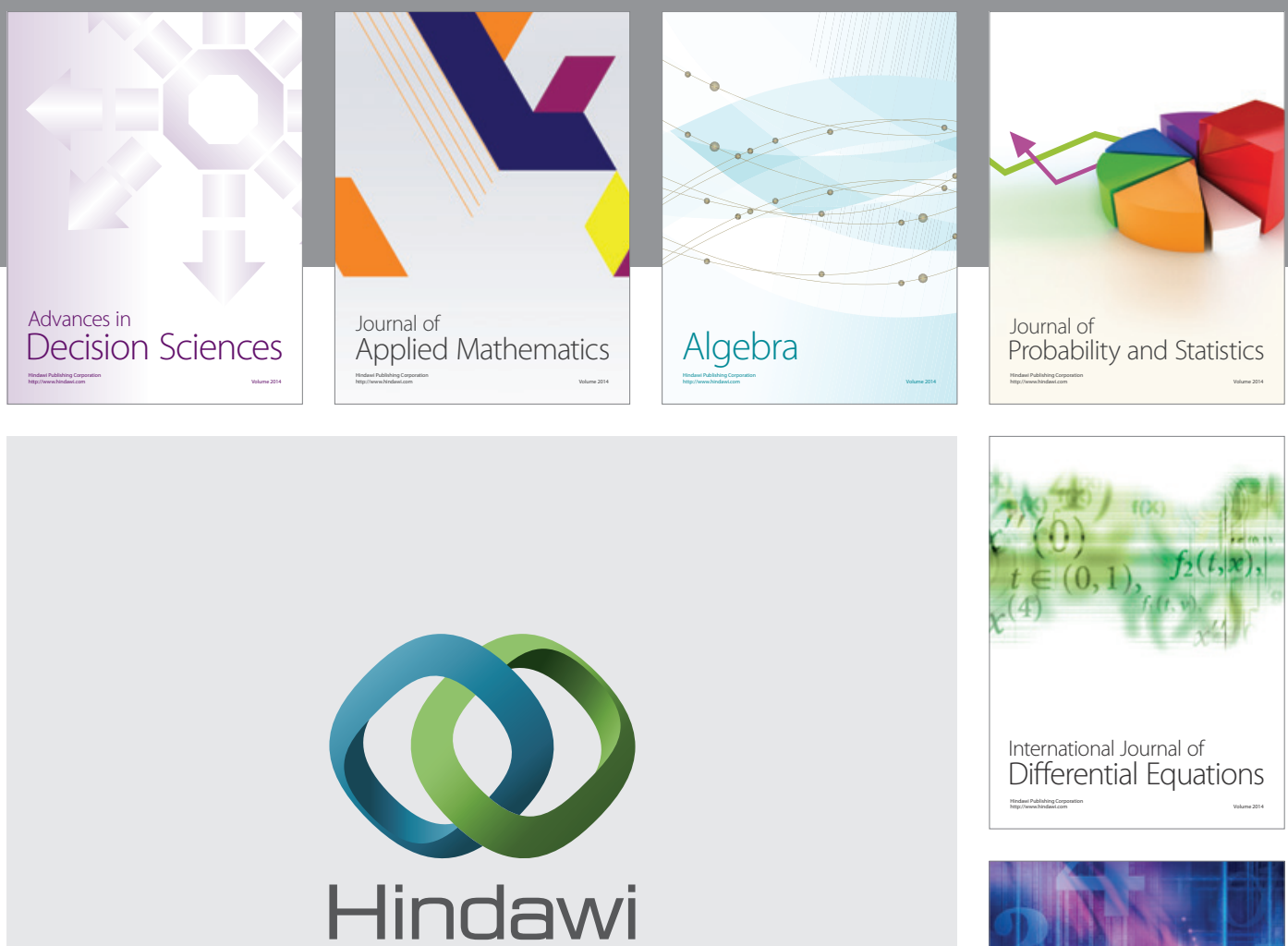

Submit your manuscripts at http://www.hindawi.com
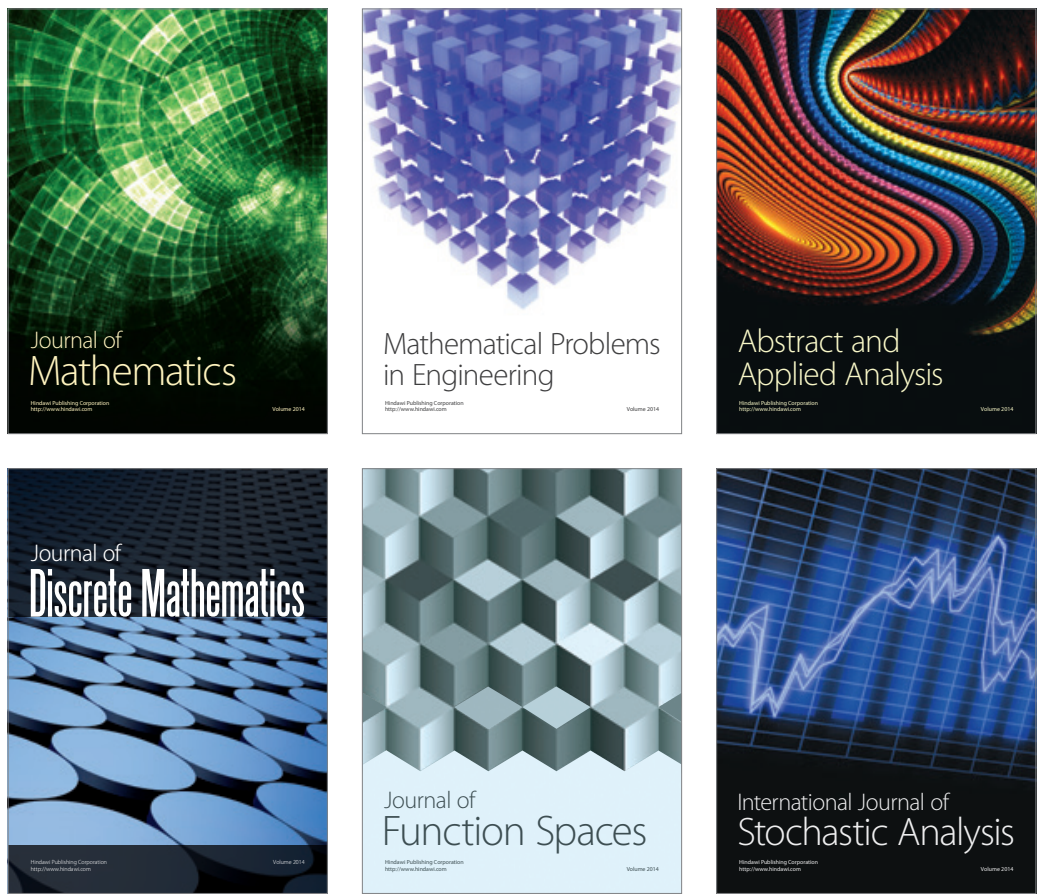

Journal of

Function Spaces

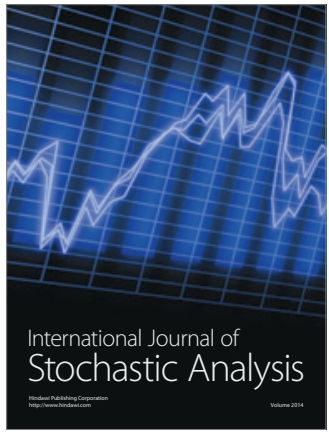

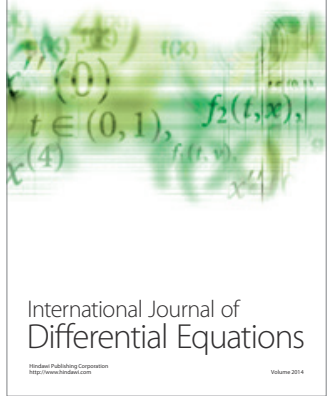
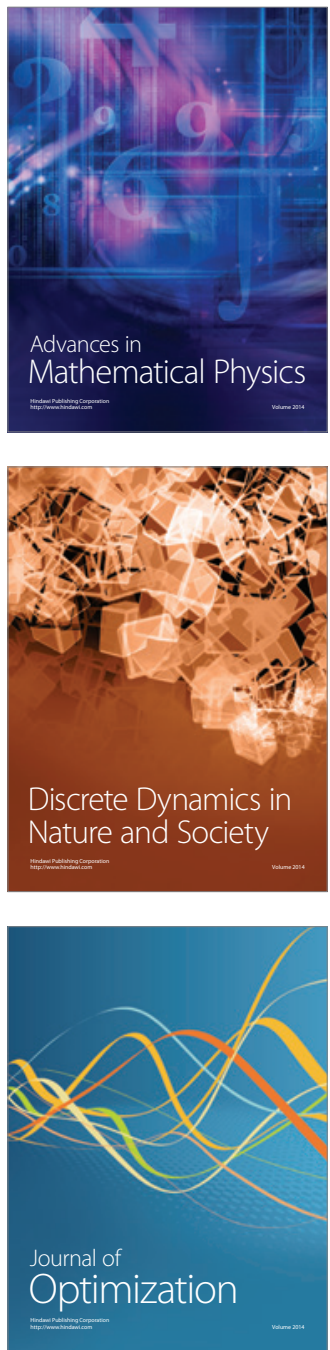